\title{
Original
}

\section{Thermal Stresses in Functionally Gradient Materials}

by

Naotake NoDA ${ }^{\dagger}$

\begin{abstract}
The thermal stresses problems of functionally gradient materials as one of the advanced high-temperature materials capable of withstanding the extreme temperature environments are discussed. One of the most important problems of thermal stresses in the functionally gradient materials is how to decrease thermal stresses. An optimal composition problems of the materials in order to decrease thermal stresses and thermal stress ploblems in the materials with a crack by fracture mechanics are treated in detail.
\end{abstract}

Key Words: Thermal Stress, Functionally Gradient Materials, Fracture Mechanics, Optimal Design, Stress Singularity

\section{INTRODUCTION}

Structural components and/or mechanical element, such as pressure vessels and pipes in nuclear reactors, chemical plants, and high-speed aircraft etc., are subjected to thermal loads due to high temperature, high gradient temperature and cyclical changes of temperature, etc. In recent year, the space planes, ultra/super/hypersonic air plants as the supersonic transport, and nuclear fusuion reactor etc., have been examined and planned in each country. These components and system are subjected to ultra-high temperature, ultra-high gradient temperature and cyclical changes of ultra-high temperature, etc. The advanced high-temperature materials capable of withstanding the extreme temperature environments have been studied in each country. The functionally gradient materials (Yamanouchi et al. $1990^{1}$, Holt et al. $1993^{2)}$ ) as one of the materials have been studying in order to decrease thermal stresses and to increase the effect of protection from heat.

One of the most important problems of the functionally gradient materials is how to decrease thermal stresses. In this paper, the current state of thermal stress problems in functionally gradient materials, an optimal composition problems of the materials in order to decrease thermal stresses and consideration of thermal stress problems in the materials by fracture mechanics are discussed. A transient thermal stresses in a plate of the materials due to one cyclic heating-cooling and stress intensity factor of a plate with a crack of the materials are treated.

\footnotetext{
Received January 27, 1955

$\dagger$ Mechanical Engineering, Faculty of Engineering

Shizuoka University

Johoku 3-5-1, Hamamatsu, 432 JAPAN
} 


\section{STEADY THERMAL STRESSES}

\section{1 Steady Thermal Stresses in a Functionally Gradient Plate}

Let us consider the steady thermal stresses in a functionally gradient plate (FGP) with thickness $l$ in figure 1 . The heat conduction equation is

$$
\frac{d}{d x}\left\{\lambda(T, x) \frac{d T}{d x}\right\}=0
$$

where $T$ is the temperature. The initial and boundary conditions are given by

$$
\begin{array}{lll}
T=T_{0}, & \text { at } & t=0 \\
T=T_{a}, & \text { on } & x=0 \\
T=T_{b}, & \text { on } & x=l
\end{array}
$$

Then, the temoerature $T$ is

$$
\mathrm{T}=T_{b}-\left(T_{a}-T_{b}\right)\left\{1-\int_{0}^{x} \frac{d x}{\lambda(x)} / \int_{0}^{x} \frac{\mathrm{dx}}{\lambda(x)}\right\}
$$

The thermal stress is

$$
\sigma(\boldsymbol{x})=\frac{\boldsymbol{E}(\boldsymbol{x})}{1-\nu(\boldsymbol{x})}\left[\varepsilon_{0}+\frac{\boldsymbol{l}}{\rho} \boldsymbol{x}-\alpha\left\{\mathbf{T}(\boldsymbol{x})-\mathbf{T}_{\mathbf{0}}\right\}\right]
$$

The boundary conditions are

$$
\begin{array}{ll}
\text { [1] free expansion and free bending } & \int_{0}^{l} \sigma d x=0, \quad \int_{0}^{l} \sigma x d x=0 \\
{[2] \text { free expansion and restrained bending }} & \int_{0}^{1} \sigma d x=0, \frac{1}{\rho}=0
\end{array}
$$

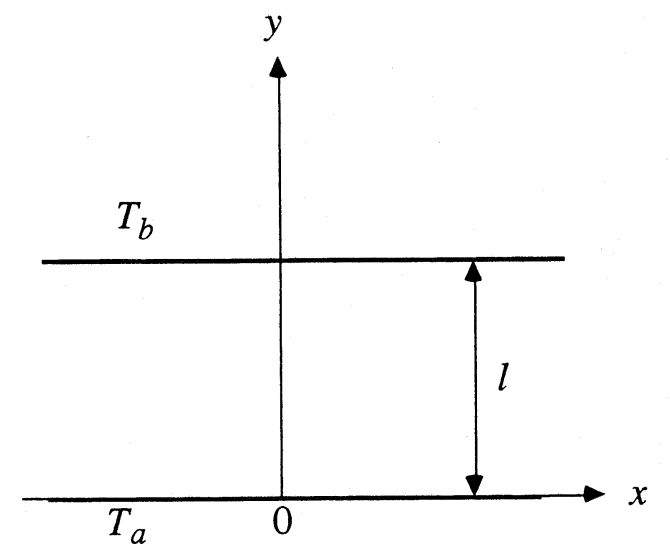

Figure 1 Functionally gradient plate 
[3] restrained expansion and free bending

$$
\begin{aligned}
& \varepsilon_{0}=0, \quad \int_{0}^{l} \sigma x d x=0 \\
& \varepsilon_{0}=0, \quad \frac{1}{\rho}=0
\end{aligned}
$$

We can obtain the thermal stresses from eqs. (2.5) and (2.6)

$$
\sigma(x)=\left(T_{b}-T_{0}\right) \sigma_{1}+\left(T_{a}-T_{b}\right) \sigma_{2}
$$

where $\lambda:$ the thermal conductivity, $l:$ the thickness of the plate, $\alpha:$ the linear thermal expansion, $\rho$ : the radius of curvature, $\varepsilon_{0}:$ the strain at $x=0, E$ : Young's modulus, $\nu$ : Poisson's ratio.

\section{2 Material Properties}

We consider the FGP of a ceramics $\left(\mathrm{ZrO}_{2}\right)$ and a metal (Ti-6A1-4V). The temperature dependency of the materials are shown in figure 2 . The full line denotes the experimental expressions deter-

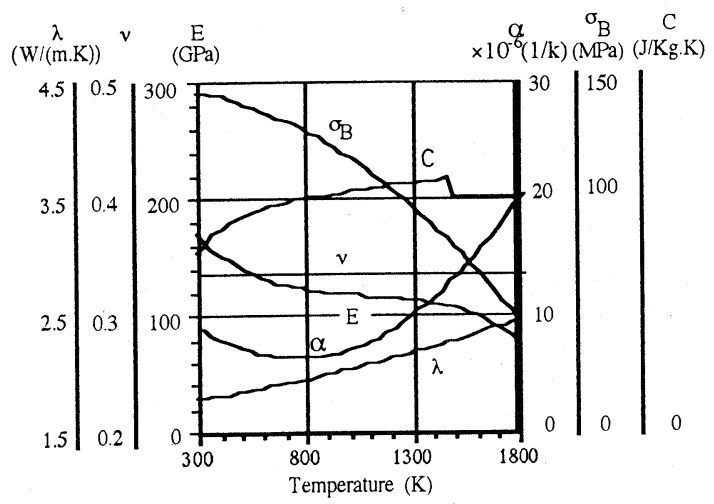

(a) $\mathrm{ZrO}_{2}$

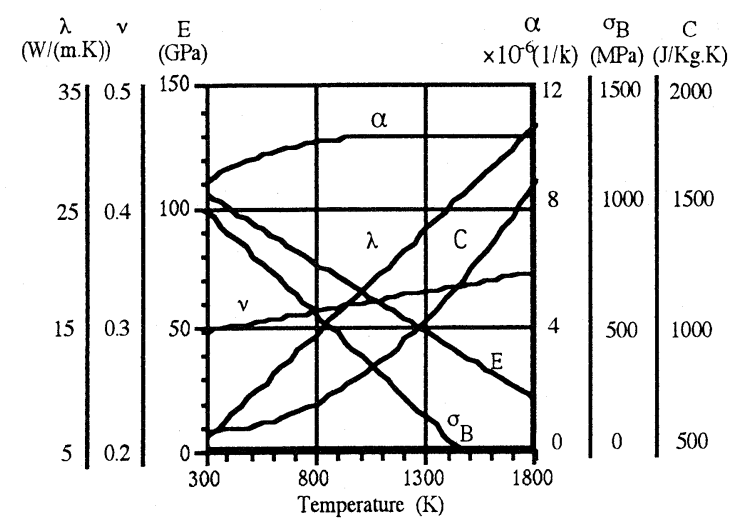

(b) Ti-6Al-4V

Figure 2 Temperature dependency of material properties 
mined from the experimental data (Cubberly $1989^{3)}$, Touloukian $1973^{4)}$ ) by least square method.

The material properties the FGM with the porosity made by the composition of the ceramics and the metal may be expressed (Kingery etc $1976^{5)}$ and Kondo $1986^{6)}$ ) as :

$$
\begin{gathered}
\lambda=\frac{\lambda_{0}\left\{\left(1-P^{2 / 3}\right)+P^{2 / 3} \lambda_{g}\right\}}{\boldsymbol{P}^{1 / 3} \lambda_{0}+\left(1-P^{1 / 3}\right)\left\{\left(1-P^{2 / 3}\right) \lambda_{0}+P^{2 / 3} \lambda_{g}\right.}, \quad E=E_{0} \frac{1-P}{1+\frac{P\left(5+\nu_{0}\right)\left(37-8 \nu_{0}\right)}{8\left(1+\nu_{0}\right)\left(23+8 \nu_{0}\right)}} \\
\alpha=\alpha_{0}, \quad \nu=\nu_{0}, \quad \sigma_{B t}=\sigma_{B t 0}(1-P), \quad \sigma_{B c}=\sigma_{B c 0}(1-P)
\end{gathered}
$$

where $P$ is the porosity, $\lambda_{g}$ is the thermal conductivity of air, and

$$
\begin{aligned}
& \lambda_{0}=k_{c}\left\{1+\frac{3\left(\lambda_{m}-\lambda_{c}\right) V_{m}}{3 \lambda_{c}+\left(\lambda_{m}-\lambda_{c}\right)\left(1-V_{m}\right)}\right\}, \quad E_{0}=E_{c}\left\{1+\frac{E_{c}+\left(E_{m}-E_{c}\right) V_{m}^{2 / 3}}{E_{c}+\left(E_{m}-E_{c}\right)\left(V_{m}^{2 / 3}-V_{m}\right)}\right\} \\
& \alpha_{0}=k_{c}\left\{1+\frac{\alpha_{m} V_{m} E_{m} /\left(1-\nu_{m}\right)+\alpha_{c}\left(1-V_{m}\right) E_{c} /\left(1-\nu_{c}\right)}{V_{m} E_{m} /\left(1-\nu_{m}\right)+\left(1-V_{m}\right) E_{c} /\left(1-\nu_{c}\right)}\right\}, \quad \nu_{0}=\nu_{m} V_{m}+\nu_{c}\left(1-V_{m}\right) \\
& \sigma_{B t 0}=\sigma_{B t m} V_{m}+\sigma_{B t c}\left(1-V_{m}\right), \quad \sigma_{B c 0}=\sigma_{B c m} V_{m}+\sigma_{B c t c}\left(1-V_{m}\right)
\end{aligned}
$$

in which $V_{m}$ denotes the volumetric ratio of the metal.

The volumetric ratio and the porosity are taken as

$$
V_{m}=x^{m}, \quad P=A x^{n}\left(1-x^{k}\right)
$$

where

$$
\left(\frac{n+\kappa}{n}\right)^{n} /\left\{1-\left(\frac{\mathrm{n}}{n+\kappa}\right)^{k}\right\} \geqq A \geqq \mathbf{0}
$$

and $m, n, \kappa$ are arbitrary constants. The volumetric ratio of the metal is shown in figure 3 . When $m=1$, the variation of the composition of ceramics and metal linear, and the metal is rich when $m<1$

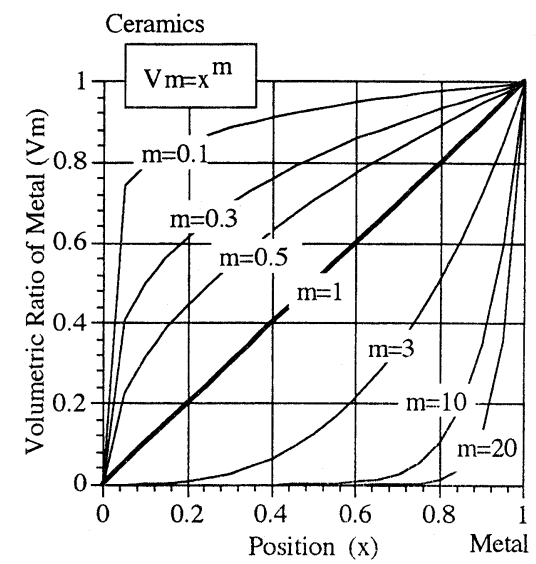

Figure 3 Volumetric ratio of metal 
and vice versa.

Next, we define two parameters. One is a safety factor $f$ defined by

$$
\begin{aligned}
\text { safety factor } \quad \boldsymbol{f}(\boldsymbol{x})=\operatorname{Min}\left(\boldsymbol{f}_{t}, \boldsymbol{f}_{\boldsymbol{c}}\right) \\
\boldsymbol{f}_{t}(\boldsymbol{x})=\sigma_{B t}(\boldsymbol{x}) / \sigma(\boldsymbol{x}), \text { for } \sigma(\boldsymbol{x}) \geqq \mathbf{0} \\
\boldsymbol{f}_{\boldsymbol{c}}(\boldsymbol{x})=\sigma_{B \boldsymbol{c}}(\boldsymbol{x}) / \sigma(\boldsymbol{x}), \text { for } \quad \sigma(\boldsymbol{x}) \leqq \mathbf{0}
\end{aligned}
$$

and the other is non-destructive thickness ratio $x_{f \geq 1}$ defined by

$$
x_{f \geq 1}=\text { thickness without destruction / thickness of the plate }
$$

\section{3 Condition of zero thermal stress}

The condition of zero thermal stress condition for the one dimensional problem can be expressed by $\left(\right.$ Noda, $1986^{7)}$ )

$$
\int_{T_{0}}^{T} \alpha\left(T^{\prime}, x\right) d T^{\prime}=d+e x
$$

That is, the thermal stress is free in the limited case that the free thermal expansion is a linear function of $x$.

\section{4 Thermal stresses in the plate}

Let us consider the steady thermal stress in the plate for three standpoints :

[Case 1] An isotropic homogeneous plate

We consider an isotropic homogeneous plate. The free thermal expansion can be expressed by a linear function of $x$ in this case, so that the free thermal expansion agrees with the condition (2.13) of zero thermal stress. Then, thermal stress does not occur.

$$
\sigma=\mathbf{0}
$$

\section{[Case 2] An isotropic homogeneous plate with two layers}

We consider an isotropic homogeneous plate with two layers. The thermal stresses on the boundary and bonded surface for various thickness ratios $\left(h_{1} / h\right)$ are shown in figure 4 . We can find three numerical result from the figures.

(1) The large tensile thermal stresses are produced on the bonded surface.

(2) The thermal stresses in the plate with temperature dependent material properties are larger than one with the temperature independent material properties.

(3) The thermal stresses decrease with increasing the thickness of the ceramics layer or of the metaric layer.

\section{[Case 3] Functionally gradient plate}

We consider a functionally gradient plate (FAP) by the composition of a ceramics $\left(\mathrm{ZrO}_{2}\right)$ and a metal (Ti-6A1-4V). The safety factors for the volumetric ratio of metal and for the ratio of porosity are shown in figure 5. We can find following results from the figures.

(1) If we can pertinently select the constants $A$ and $m$, the FGP with temperature independent prop erties not be destroyed. Because the safety factor is greater than 1 .

(2) The safety factors of the FGP with temperature dependent properties are always less than 1, so that the late will be destroyed. The maximum safety factor is 0.277 at the optimal values 
$A=2.29$ and $m=30$.

(3) When a new ratio of porosity $\left(P=A x^{n}(1-x)\right)$ will be selected, the safety factor of the FGP with temperature dependent properties becomes 1.55 at $m=2.73, A=13.6$ and $n=4.8$. Then, the FAP will not be destroyed.

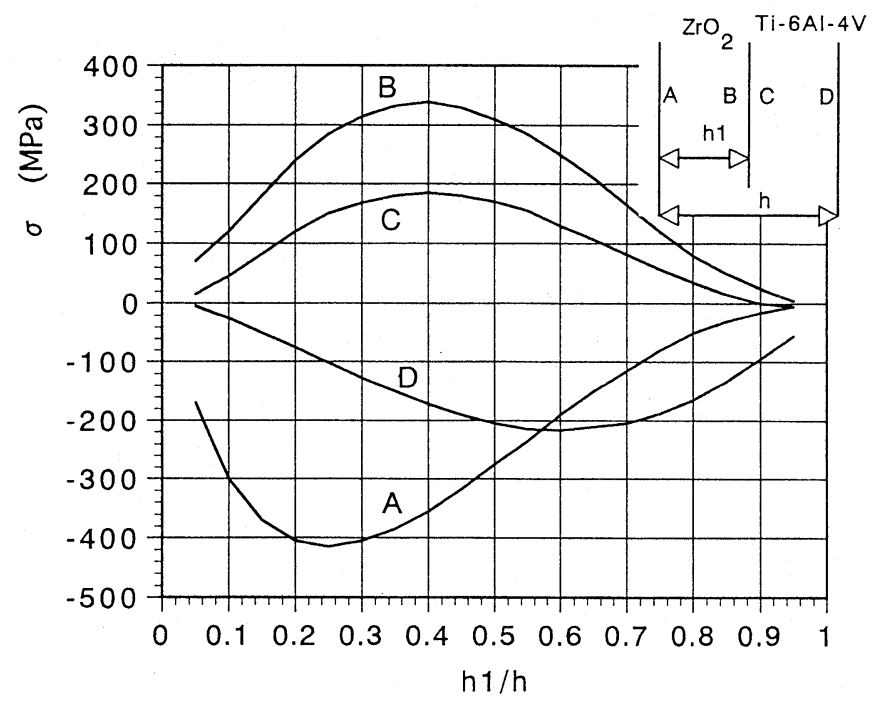

(a) temperature independent properties

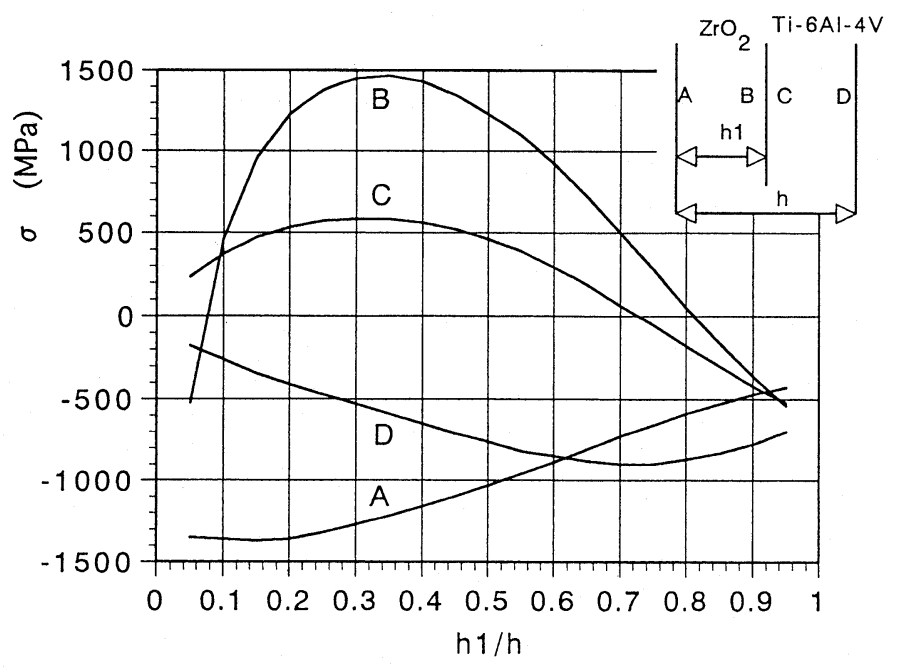

(b) temperature dependent properties

Figure 4 Effects of the thickness ratio on the thermal stresses in the plate with two layers 


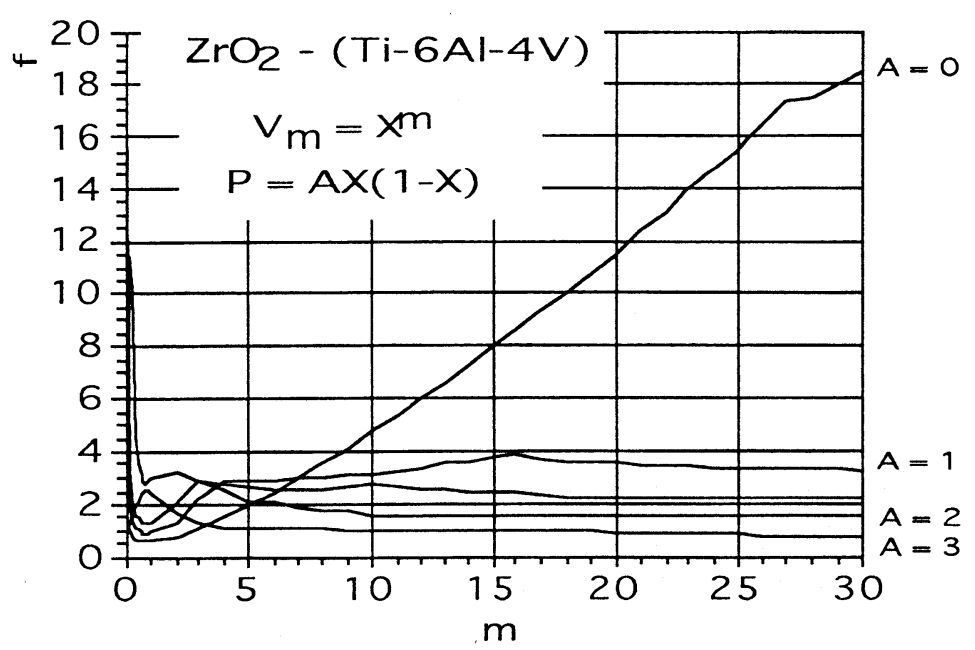

(a) temperature independent properties

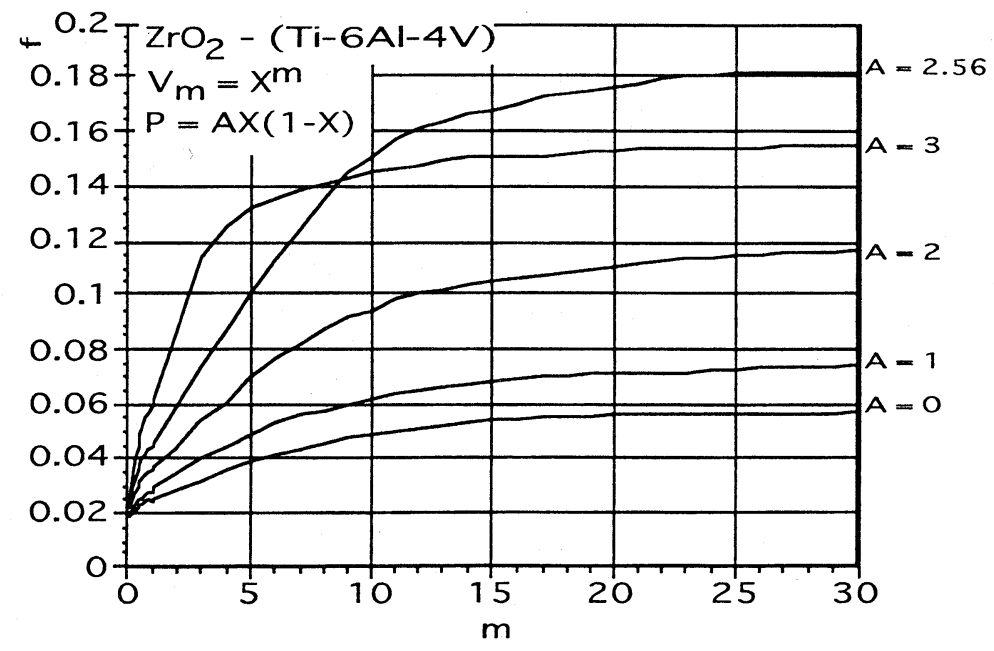

(b) temperature dependent properties

Figure 5 Effects of the constant $m$ for volumetric ratio and constant $\boldsymbol{A}$ for the porosity on the safety factor 


\section{TRANSIENT THERMAL STRESSES IN THE FGP}

\section{1 Temperature field}

Let us consider the transient thermal stresses in the FGP whose material properties are independent of temperature. The heat conduction equation of isotropic and nonhomogeneous materials is governed by

$$
c \rho \frac{\partial T}{\partial t}=\kappa \nabla^{2} T+\nabla \lambda \nabla T
$$

where $\mathrm{c}$ is the specific heat, $\rho$ is the density and $\lambda$ is the thermal conductivity. It is assumed that these material constants are continuous functions of position and $\lambda$ is continuously differentiable. $\nabla^{2}$ is the Laplacian operator and $\nabla$ is the gradient operator. Equation (3.1) reduces to for one dimensional temperature field

$$
c(\boldsymbol{x}) \rho(\boldsymbol{x}) \frac{\partial T}{\partial t}=\frac{\partial}{\partial \boldsymbol{x}}\left\{\lambda(\boldsymbol{x}) \frac{\partial T}{\partial \boldsymbol{x}}\right\}
$$

The initial and boundary conditions are given by

$$
\begin{aligned}
& T=T_{0}(x), \quad \text { at } \quad t=0 \\
& T=T_{a}, \quad \text { on } \quad x=0 \\
& T=T_{b}, \quad \text { on } \quad x=l
\end{aligned}
$$

In order to solve the heat conduction equation (3.1), we introduce a variable transformation :

$$
\xi=\int_{0}^{x} \frac{d x}{\sqrt{\kappa(x)}}
$$

where denotes the thermal diffusivity. Equation (3.1) reduces to

$$
\frac{\partial T}{\partial t}=\frac{\partial^{2} T}{\partial \xi^{2}}+\delta \phi(\xi) \frac{\partial T}{\partial \xi}
$$

where

$$
\delta \phi(\xi) \equiv \frac{d}{d \xi} \ln \sqrt{c(\xi) \rho(\xi) \kappa(\xi)}
$$

Using the perturbation method :

$$
T(x, t)=\sum_{n=0}^{\infty} \delta^{n} T^{(n)}(x, t)
$$

and the Laplace transformation :

$$
T^{*}(x, s)=\int_{0}^{\infty} e^{-s t} T(x, t) d t
$$


we can obtain the general solution of the temperature in the Laplace domain

$$
\begin{aligned}
& T^{*(0)}=A_{0} \cosh \sqrt{s} \xi+B_{0} \sinh \sqrt{s} \xi-\frac{1}{\sqrt{s}} \int_{0}^{\xi} T_{0}(\eta) \sinh \sqrt{s}(\xi-\eta) d \eta \quad \text { for } n=0 \\
& T^{*(n)}=A_{n} \cosh \sqrt{s} \xi+B_{n} \sinh \sqrt{s} \xi-\frac{1}{\sqrt{s}} \int_{0}^{\xi} \phi(\eta) \frac{\mathrm{dT}^{*(n-1)}}{d \eta} \sinh \sqrt{s}(\xi-\eta) d \eta \quad \text { for } n \geqq 0
\end{aligned}
$$

where $A_{0}, B_{0}, A_{n}$, and $B_{n}$ are unknown coefficients to be determined from the boundary conditions (3.3). After determination of the unknown coefficients, the temperature field in the Laplace transformed domain is given by

$$
T^{*}(x, s)=\sum_{i=0}^{\infty} \delta^{i} T^{*(i)}(x, s)
$$

where

$$
\begin{aligned}
T^{*(0)}= & \frac{1}{s \sinh \sqrt{s} \zeta}\left\{T_{a} \sinh \sqrt{s}(\zeta-\xi)+T_{b} \sinh \sqrt{s} \xi\right\} \\
\delta T^{*(1)}= & \frac{\sinh \sqrt{s} \xi}{\sqrt{s} \sinh ^{2} \sqrt{s} \zeta} \int_{0}^{\zeta} \ln \sqrt{c \rho \kappa}\left\{T_{b} \cosh \sqrt{s}(\zeta-2 \eta)-T_{a} \cosh 2 \sqrt{s}(\zeta-\eta)\right\} d \eta \\
& +\frac{1}{\sqrt{s} \sinh \sqrt{s} \zeta} \int_{0}^{\xi} \ln \sqrt{c \rho \kappa}\left\{T_{b} \cosh \sqrt{s}(\zeta-2 \eta)-T_{a} \cosh 2 \sqrt{s}(\xi+\zeta-2 \eta)\right\} d \eta \\
\delta^{n} T^{*(n)} & =\frac{\sinh \sqrt{s} \xi}{\sqrt{s} \sinh \sqrt{s} \zeta} \int_{0}^{\zeta} \ln \sqrt{c \rho \kappa} \frac{d}{d \eta}\left[\sinh \sqrt{s}(\zeta-\eta) \frac{d}{d \eta}\left\{\delta^{n-1} T^{*(n-1)}\right\}\right\} d \eta \\
& +\frac{1}{\sqrt{s}} \int_{0}^{\eta} \ln \sqrt{c \rho \kappa} \frac{d}{d \eta}\left[\sinh \sqrt{s}(\xi-\eta) \frac{d}{d \eta}\left\{\delta^{n-1} T^{*(n-1)}\right\}\right\} d \eta
\end{aligned}
$$

in which

$$
\zeta=\int_{0}^{l} \frac{d x}{\sqrt{\kappa(x)}}
$$

Next, performing the inverse Laplace transformation to (3.11)

$$
T(x, t)=\frac{1}{2 \pi i} \int_{0}^{\infty} e^{t s} T^{*}(x, s) d s
$$

we can obtain the temperature field

$$
T(x, t)=\sum_{i=0}^{\infty} \delta^{i} T^{(i)}(x, t)
$$


Table 1 Comparison of Solution at Transient State

(a) $t^{\prime}=10^{-2}$

\begin{tabular}{|c|l|l|l|l|}
\hline & Analy. & \multicolumn{3}{|c|}{ Perturbation Tech. } \\
\hline $\boldsymbol{x} / \boldsymbol{l}$ & \multicolumn{1}{|c|}{$\boldsymbol{T}$} & $\boldsymbol{N}=0$ & $\boldsymbol{N}=1$ & $\boldsymbol{N}=2$ \\
\hline 0.0 & 0.5 & 0.5 & 0.5 & 0.5 \\
0.1 & 0.2399 & 0.2341 & 0.2399 & 0.2399 \\
0.2 & 0.0716 & 0.0681 & 0.0716 & 0.0716 \\
0.3 & 0.0117 & 0.0108 & 0.0116 & 0.0117 \\
0.4 & 0.0009 & 0.0008 & 0.0009 & 0.0009 \\
0.5 & 0.0 & 0.0 & 0.0 & 0.0 \\
0.6 & 0.0 & 0.0 & 0.0 & 0.0 \\
0.7 & 0.0003 & 0.0003 & 0.0003 & 0.0003 \\
0.8 & 0.0140 & 0.0152 & 0.0139 & 0.0140 \\
0.9 & 0.2567 & 0.2687 & 0.2565 & 0.2565 \\
1.0 & 1.5 & 1.5 & 1.5 & 1.5 \\
\hline
\end{tabular}

(b) $t^{\prime}=10$

\begin{tabular}{|l|l|l|l|l|}
\hline & Analy. & \multicolumn{3}{|c|}{ Perturbation Tech. } \\
\hline $\boldsymbol{x} / \boldsymbol{l}$ & \multicolumn{1}{|c|}{$\boldsymbol{T}$} & $\boldsymbol{N}=0$ & $\boldsymbol{N}=1$ & $\boldsymbol{N}=2$ \\
\hline 0.0 & 0.5 & 0.5 & 0.5 & 0.5 \\
0.1 & 0.5523 & 0.5724 & 0.5501 & 0.5523 \\
0.2 & 0.6103 & 0.6488 & 0.6068 & 0.6102 \\
0.3 & 0.6752 & 0.7298 & 0.6712 & 0.6749 \\
0.4 & 0.7483 & 0.8161 & 0.7447 & 0.7478 \\
0.5 & 0.8313 & 0.9084 & 0.8288 & 0.8307 \\
0.6 & 0.9264 & 1.0077 & 0.9256 & 0.9257 \\
0.7 & 1.0366 & 1.1151 & 1.0376 & 1.0359 \\
0.8 & 1.1653 & 1.2319 & 1.1679 & 1.1648 \\
0.9 & 1.3176 & 1.3597 & 1.3204 & 1.3174 \\
1.0 & 1.5 & 1.5 & 1.5 & 1.5 \\
\hline
\end{tabular}

in which

$$
\begin{aligned}
T^{(0)}= & T_{a}-\frac{\xi}{\zeta}\left(T_{a}-T_{b}\right)-\sum_{i=0}^{\infty} \frac{2}{i \pi}\left\{T_{a}-(-1)^{i} T_{b}\right\} \sin \left(i \pi \frac{\xi}{\zeta}\right) e^{-(i \pi / \zeta)^{2} \mathrm{t}} \\
\delta T^{(1)} & =\frac{T_{a}-T_{b}}{\zeta}\left(\int_{0}^{\xi} \ln \sqrt{c \rho \kappa} d \eta-\frac{\xi}{\zeta} \int_{0}^{\zeta} \ln \sqrt{c \rho \kappa} d \eta\right) \\
& -\frac{2}{\zeta} \sum_{i=1}^{\infty}\left[\left\{2 T_{a}-(-1)^{i} T_{b}\right\} \sin \left(i \pi \frac{\xi}{\zeta}\right) \int_{0}^{\xi} \ln \sqrt{c \rho \kappa} \sin \left(2 i \pi \frac{\eta}{\zeta}\right) d \eta\right. \\
& +\left\{T_{a}-(-1)^{i} T_{b}\right\}\left\{-\int_{0}^{\xi} \ln \sqrt{c \rho \kappa} \cos \left(i \pi \frac{\xi-2 \eta}{\zeta}\right) d \eta\right. \\
& +\cos \left(i \pi \frac{\xi}{\zeta}\right) \int_{0}^{\zeta} \frac{\xi}{\zeta} \ln \sqrt{c \rho \kappa} \cos \left(i \pi \frac{\eta}{\zeta}\right) d \eta-2 \sin \left(i \pi \frac{\xi}{\zeta}\right) \int_{0}^{\zeta} \frac{\eta}{\zeta} \ln \sqrt{c \rho \kappa} \sin \left(2 i \pi \frac{\eta}{\zeta}\right) d \eta \\
& \left.\left.-\frac{2 i \pi t}{\zeta} \sin \left(i \pi \frac{\xi}{\zeta}\right) \int_{0}^{\zeta} \ln \sqrt{c \rho \kappa} \cos \left(2 i \pi \frac{\eta}{\zeta}\right) d \eta\right\}\right] e^{-(i \pi / \zeta)^{2} \mathrm{t}} \\
& \delta^{2} \mathbf{T}^{(2)}=\ldots \ldots \ldots \ldots \ldots . . . . .
\end{aligned}
$$

Let us discuss the accuracy of this method. When a material property of the FGP may be approximately expressed as

$$
c \rho \lambda=\exp [2(c \xi+d)]
$$

the profile of the material property with $c=-0.9560$ and $d=0$ in (3.18) is good agreement of one of the FGP with $M=1$ and $A=0$. In this case, we can get theoretical solution for the heterogeneous materials as follows : 


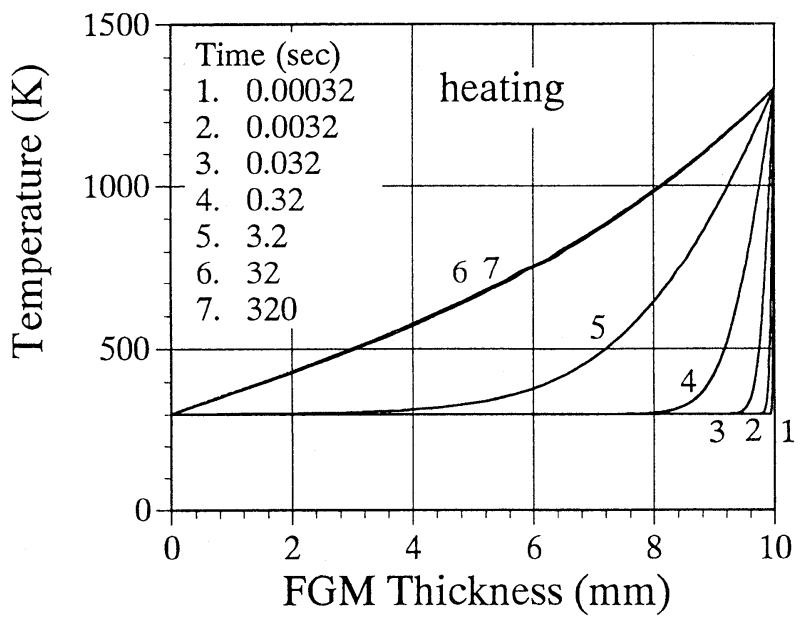

(a) heating

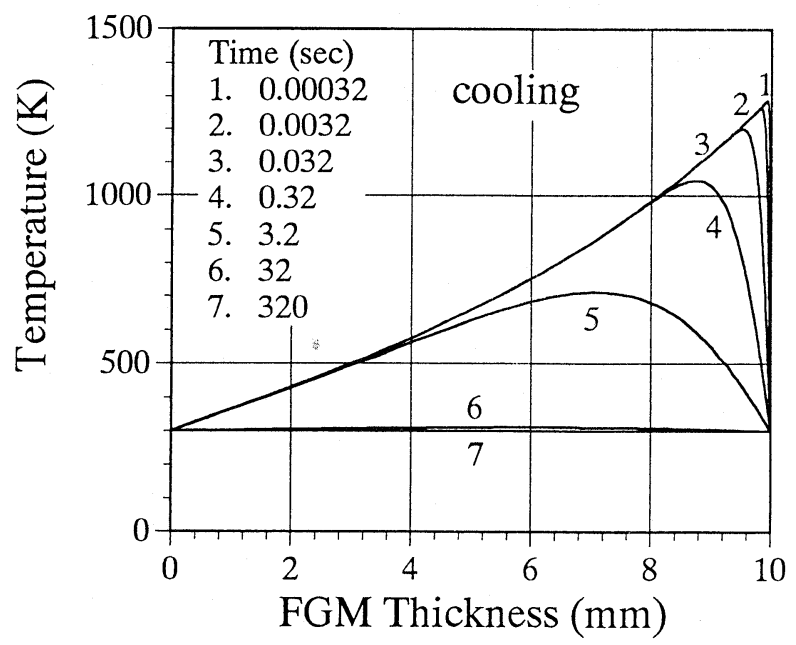

(b) cooling

Figure 6 Transient temperature distribution

$$
\begin{aligned}
T & =\frac{\exp (-c \xi / 2)}{\sinh c \eta}\left[T_{b} e^{-c \xi / 2} \sinh c \xi+T_{a} \sinh c(\xi-\eta)\right] \\
& +\sum_{m=1}^{\infty} \frac{2 m \pi}{(c \eta)^{2}+(m \pi)^{2}} e^{-c \xi / 2}\left[(-1)^{m} T_{b} e^{c \xi / 2}-T_{a}\right] \exp \left\{-\left[c^{2}+(m \pi / \eta)^{2}\right] \kappa_{b} t / l^{2}\right\}
\end{aligned}
$$




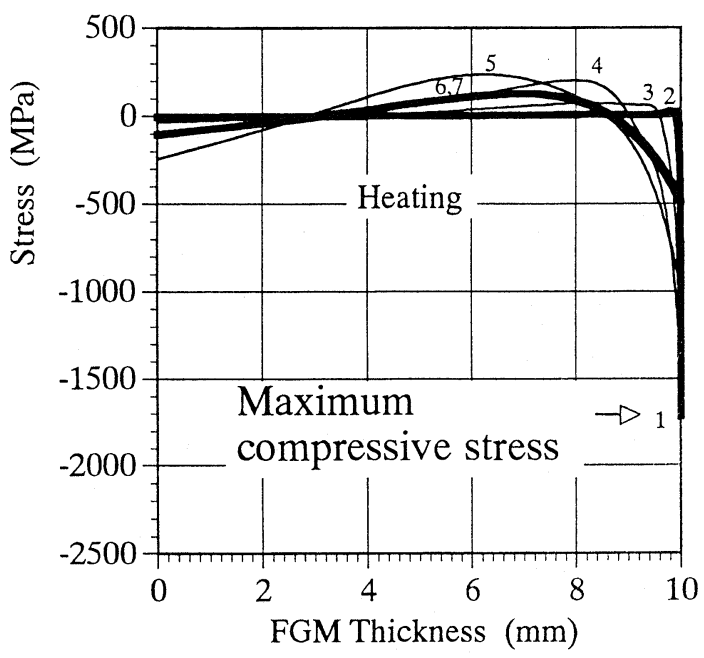

(a) heating

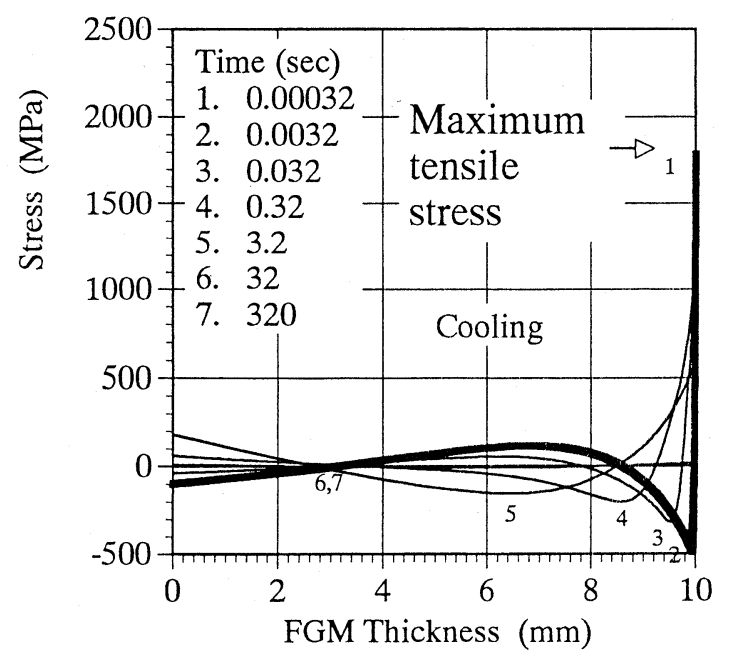

(b) cooling

Figure 7 Transient Thermal Stress Distribution

Table 1 shows the comparison of the temperature between the analytical solution (3.21) and the perturbation solution (3.18) with the truncated number $N=0,1,2$, when an each surface of the plate with the zero initial temperature is heated to $T_{a}=0.5$ and $T_{b}=1.5$. At $t^{\prime}\left(=\kappa_{b} t / l^{2}\right)=10^{-2}$ and $t^{\prime}=10$, the perturbation solutions for $N=2$ are good agreement with the analytical results. The maximum error of the perturbation solution for $N=2$ at $t^{\prime}=10$ was only $0.07 \%$. The temperature (3.16) obtained from proposal technique has with good accuracy. 
3. 2 Thermal stresses in the FGP subjected to one cycle heating with heating and cooling

\section{2. 1 Temperature}

We assume that the FGP is suddenly heated from the initial temperature $T_{0}=300 \mathrm{~K}$ to $T_{a}=770$ $\mathrm{K}, T_{b}=1680 \mathrm{~K}$, and after steady state is estimated, the temperatures on the boundary surfaces of the FGP are suddenly cooled to the initial temperature $T_{0}=300 \mathrm{~K}$. The combination of the above temperatures is one of the combinations that we can design non-damage FGM plate at the steady state for variable mechanical boundary conditions (2.6). Figure 6(a) shows the temperature fields at heating, while figure 6 (b) shows the temperature at cooling. In the both cases of heating and cooling, the temperature suddenly changes near the both boundary

\section{2. 2 Thermal Stresses}

The thermal stress is given by

$$
\sigma(\boldsymbol{x})=\frac{\boldsymbol{E}(\boldsymbol{x})}{1-\nu(\boldsymbol{x})}\left[\varepsilon_{0}+\frac{\boldsymbol{l}}{\rho} \boldsymbol{x}-\alpha\left\{\boldsymbol{T}(\boldsymbol{x})-\boldsymbol{T}_{\mathbf{0}}\right\}\right]
$$

The strain and the curvature on a neutral plane can be determined by the boundary conditions (2.6).

Figure 7 shows the transient thermal stresses in the FGP with the boundary condition (2.6-4). In the figure $7(\mathrm{a})$, the large compressive stress occurs on the ceramics surface at an earlier stage of the heating, the stress suddenly decreases with a short distance from ceramics surface, and the tensile stress appears inside of the plate. The large compressive stress on the ceramic surface rapidly decreases with time and the steady thermal stress is very small than the transient thermal stresses at eairlier stage. On the other hand, the large tensile stress occurs on the ceramics surface whose tensile strength is very weak at the earlier stage of cooling in the figure $7(b)$. The large tensile stress on the surface rapidly dereases with time. Such large tensile stress may be the important factor to design the FGM. Because such large tensile stress will give the damage in the FGM.

If the thermal stress is larger than the strength, the FGP will be destroyed. We can easily find from the figure that the crack length ratio (= crack length/thickness of plate) is about $10 \%$. Then, the damage zone in the FGM is restricted within the very thin layer from the ceramics surface.

\section{3 Thermal Stresses in the FGP with temperature dependent properties}

Let us consider the thermoelastic stress in the FGP with temperature dependent material properties. We assume that the FGP is suddenly heated from the initial temperature $T_{0}=300 \mathrm{~K}$ to $T_{a}=$ $300 \mathrm{~K}, T_{b}=1300 \mathrm{~K}$, and after steady state is estimated, the temperatures on the boundary surfaces of the FGP are suddenly cooled to the initial temperature $T_{0}=300 \mathrm{~K}$. In this case, Numerical results are obtained by the finite element method.

We find in the section 3.2.2 that the damage zone in the FGM is restricted within the very thin layer from the ceramics surface. Then, let consider how to distribute the volumetric ratio of composition and the ratio of porosity in order minimize the damage zone of the FGP. Figure 8 shows the thermal stress on the ceramics surface versus the coefficient of the volumetric ratio of composition.

Symbol $\boldsymbol{\Delta}$ denotes the maximum tensile stress on the ceramic surface at cooling, and symbol $\bigcirc$ denotes the residual stress on the ceramics surface at steady state during heating. The maximum tensile stress decreases with increasing the compressible residual stress at the steady state. When $m=1$, that 


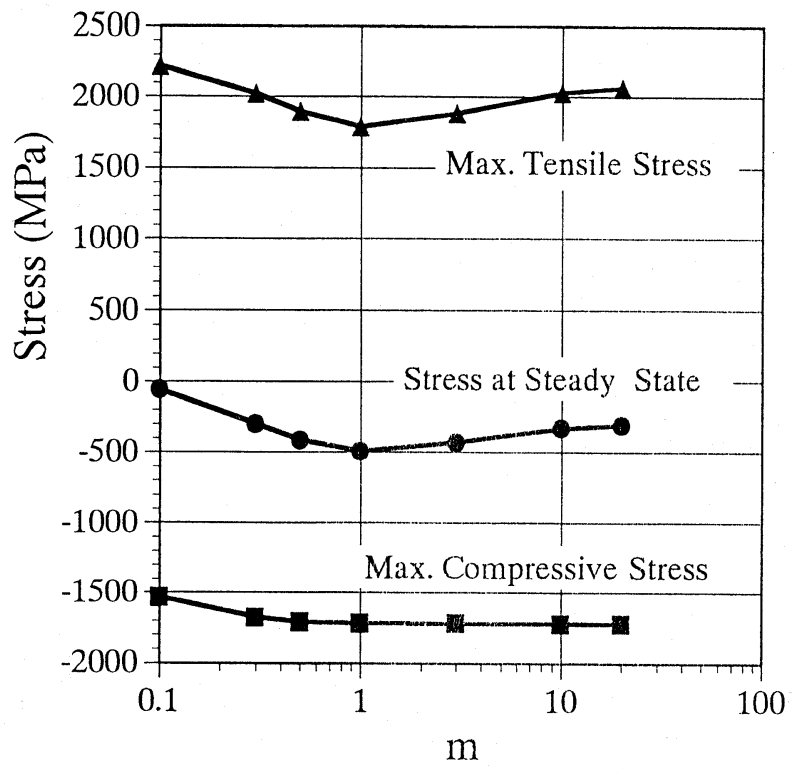

Figure 8 Thermal Stress versus $m$

is, the composition between the metal and ceramics changes lineariy, the maximum tensile stress is smallest. Otherwise, the stress at steady state occurs the maximum compression at $m=1$. Because the maximum tensile stress is given by the summation of the residual stress and the thermal stress $(-\alpha E \nabla T /(1-\nu))$ which occurs by thermal shock.

\section{THERMAL STRESSES IN THE FGP WITH A CRACK}

The stress field in the homogeneous materials with cracks is ususlly discussed by the stress intensity factor. For cracks in FGM as non-homogeneous materials, can stress field near the crack tip still be characterized by the well known K-field? Stress singularity of two dimensional non-homogeneous materials with crack is discussed in this section. The material properties are assumed to be continuous and sufficiently differentiable. The local cylindrical coordinate system $(r, \theta)$ at the crack tip is

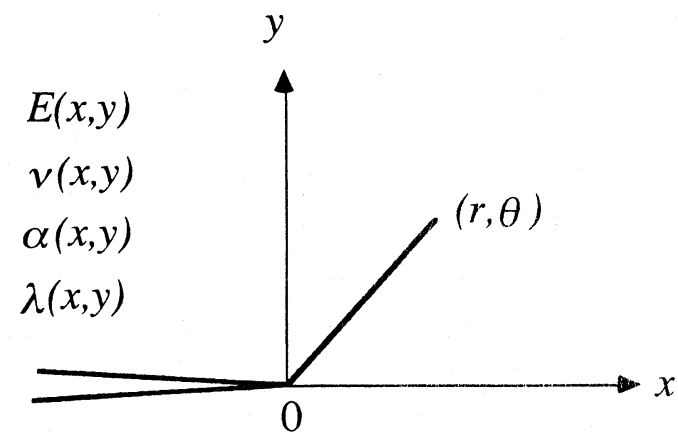

Figure 9 Local coordinate at the crack tip 
shown figure 9.

\subsection{Temperature field}

In the very near crack-tip region, the following asymptotic solution of temperature $T$ is assumed

$$
\boldsymbol{T}(\boldsymbol{r}, \theta, \boldsymbol{t})=\boldsymbol{r}^{\delta} \mathbf{\boldsymbol { N }}(\theta) \boldsymbol{f}(\boldsymbol{t})
$$

where $\delta$ is the eigenvalue and $f(t)$ is a time-dependent parameter. By substituting (4.1) into (3.1) and only taking dominant part in the equation as $r \rightarrow 0$, we have

$$
\nabla^{2} T=0
$$

The crack faces are supposed to be completely insulated, which corresponds to the following boundary conditions

$$
\frac{\partial T}{\partial \theta}=0 \quad \text { on } \quad \theta= \pm \pi
$$

The equations (4.2) and (4.3) are same equations for an isotropic materials (sih, 1965). Therefore, the crack-tip temperature field and heat fluxes are

$$
\begin{aligned}
& T(r, \theta, t)=f(t) \sqrt{2 r} \sin \frac{\theta}{2} \\
& q_{r}=-\lambda^{0} \frac{\partial T}{\partial r}=-\lambda^{0} f(t) \frac{1}{\sqrt{2 r}} \sin \frac{\theta}{2} \\
& q_{r}=-\lambda^{0} \frac{\partial T}{r \partial \theta}=-\lambda^{0} f(t) \frac{1}{\sqrt{2 r}} \cos \frac{\theta}{2}
\end{aligned}
$$

Then, the singularity and the angular distribution of the fields for FGM and the homogeneous materials are identical.

\section{2 Themoelastic field}

Thermoelastic constitutive equations are

$$
\varepsilon_{i j}=\frac{1+\nu}{E} \sigma_{i j}-\frac{\nu}{E} \sigma_{k k} \delta_{i j}+\alpha \boldsymbol{T} \delta_{i j}
$$

Under the plane stress condition, the equilibrium equations are satisfied by using the Airy stress function $F$

$$
\sigma_{x x}=\frac{\partial^{2} F}{\partial y^{2}}, \quad \sigma_{y y}=\frac{\partial^{2} F}{\partial x^{2}}, \quad \sigma_{x y}=\frac{\partial^{2} F}{\partial x \partial y}
$$

The compatibility condition is given by the Airy stress function $F$

$$
\nabla^{2}\left(\frac{1}{E} \nabla^{2} F\right)-\frac{\partial^{2}}{\partial y^{2}}\left(\frac{1+\nu}{E}\right) \frac{\partial^{2} F}{\partial x^{2}}-\frac{\partial^{2}}{\partial x^{2}}\left(\frac{1+\nu}{E}\right) \frac{\partial^{2} F}{\partial y^{2}}+\frac{\partial^{2}}{\partial x \partial y}\left(\frac{1+\nu}{E}\right) \frac{\partial^{2} F}{\partial x \partial y}+\nabla^{2}(\alpha T)=0
$$


where

$$
\nabla^{2}\left(\frac{1}{E} \nabla^{2} F\right)=\nabla^{2}\left(\frac{1}{E}\right) \nabla^{2} F+2 \nabla\left(\frac{1}{E}\right) \cdot \nabla\left(\nabla^{2} F\right)+\frac{1}{E} \nabla^{2} \nabla^{2} F
$$

Suppose the Airy stress function $F$ has following asymptotic from solution at the crack tip

$$
\boldsymbol{F}=\boldsymbol{r}^{\lambda} \mathbf{\boldsymbol { N }}(\theta, \boldsymbol{M}) \boldsymbol{g}(\boldsymbol{t})
$$

where $\lambda$ is the eigenvalue, $g(t)$ is time-dependent parameter which cannot determined by the local analysis, and $M$ is the mixture parameter defined by

$$
M=\frac{2}{\pi} \tan ^{-1}\left\{\lim _{r \rightarrow 0} \frac{\sigma_{x y}(r, 0)}{\sigma_{y y}(r, 0)}\right\}
$$

By substituting (4.9) into (3.1) and nothing temperature expression (4.4) and material properties are sufficiently differentiable, and only taking dominant part in the equation as $r \rightarrow 0$, we get the dominant part of (4.8)

$$
\nabla^{2} \nabla^{2} \boldsymbol{F}=\mathbf{0}
$$

Hence, the dominant singularity solution for FGM is still governed by the biharmonic equation which is governing equation of homogeneous materials.. Therefore, one can come to the conclusion that the singularity and the angular distribution of the crack-tip fields for FGM are identical to that in the homogeneous material.

\section{3 Thermal Stresses in the FGP with a edge crack}

Let us consider the thermoelastic stresses in a platewith a edge crack of the FGM with temperature dependent materials. We assume that the temperatures on the boundaries of the plate with a edge crack of crack length $a_{i}$ is suddenly heated from the initial temperature $T_{0}=300 \mathrm{~K}$ to $T_{a}=300 \mathrm{~K}$, $T_{b}=1300 \mathrm{~K}$, and after steady state is suddenly cooled to the initial temperature $T_{0}=300 \mathrm{~K}$ as shown in Figure 10. The material properties which are dependent on the temperature, are shown in figure 3. Numerical results are calculated for the linear composition of materials $(V=x$ and $P=0$ in $(2.10)$ ) by the finite element method.

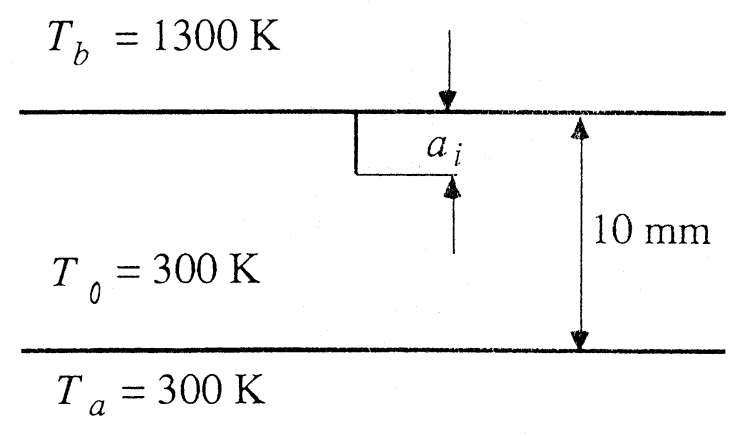

Figure 10 Local coordinate at the crack tip 
Figure 11 shows the variation of stress intensity factor (SIF) Mode I for various initial crack lengths versus time. The SIF Mode I $\left(K_{I}\right)$ occurs the largest value at very short time from cooling and it decreases with time. Figure 12 shows the crack opening displacements (COD) in the case of crack length $a_{i}=0.1 \mathrm{~mm}$. The crack does not open while heating stage, but it opens at the cooling stage. The crack open displacements reach the largest value at $t=0.032$. Figure 13 shows the crack propagation during thermal shock at the cooling stage. The fracture toughness $K_{1 c}$ (bold face in figure) is a function of position because of variation of composition in the FGP. When the $K_{I}$ of the FGP with a initial crack length $a_{i}$ is larger than $K_{1 c}$ at the initial crack length $a_{i}$, the crack propaga-

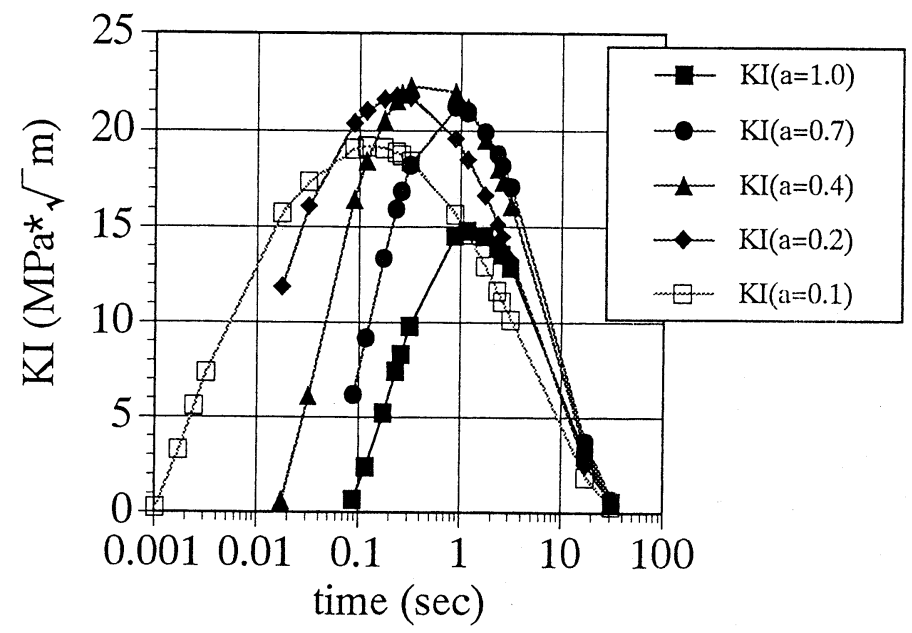

Figure 11 SIF versus time

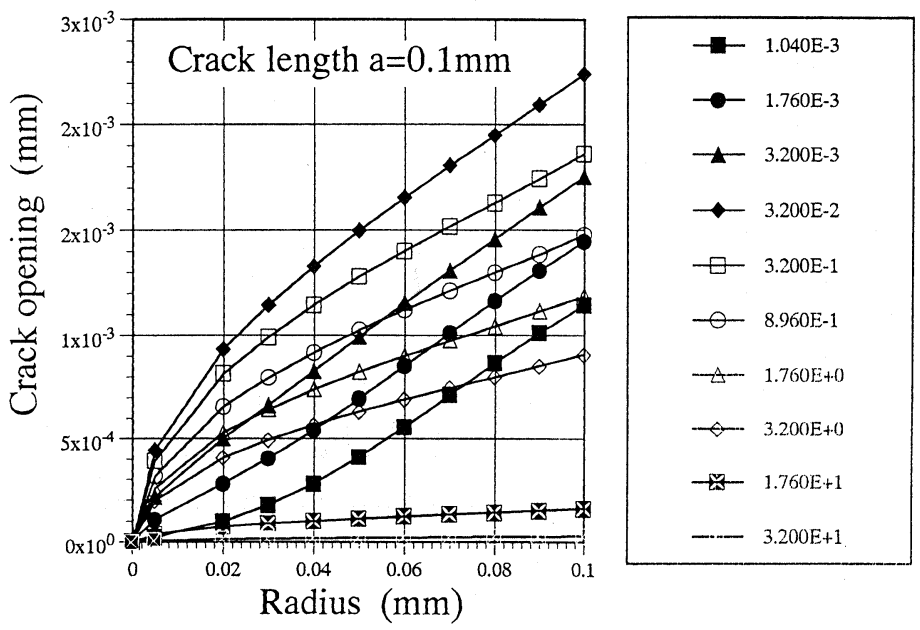

Figure 12 COD versus time 


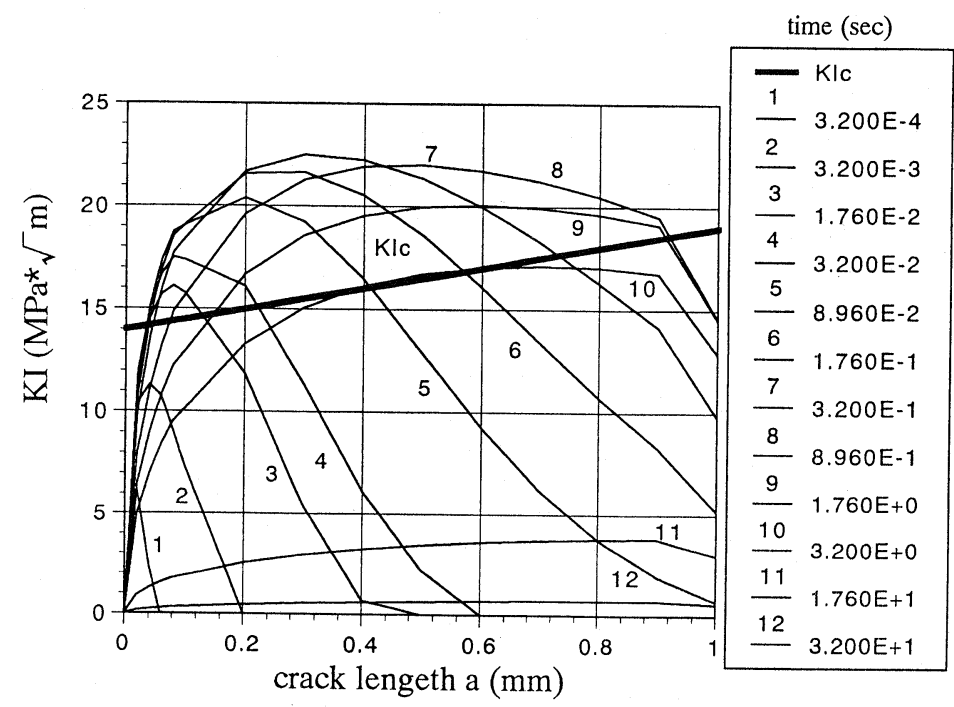

Figure 13 Crack propagation during Thermal Shock

tion occurs till the $K_{I}$ at a crack length $a$ equals $K_{1 c}$ at a crack length $a$. When a small defect on the ceramic surfase is longer than about $0.04 \mathrm{~mm}$ in this case, the crack propagates from the ceramic surface with small initial defects $a_{i}$ till $a=0.9 \mathrm{~mm}$. The unstable crack propagation occurs between $a_{i}=0.04<a<0.1 \mathrm{~mm}$ at early time during thermal shock, and after them a stable crack propagation occurs till $a=0.9 \mathrm{~mm}$.

Figure 14 shows the effect of parameter of volumetric ratio $m$ on maximum stress intensity factor $K_{1 \max }$. When $m=1$, that is, the composition of the metal and ceramics changes linearly, the maxi-

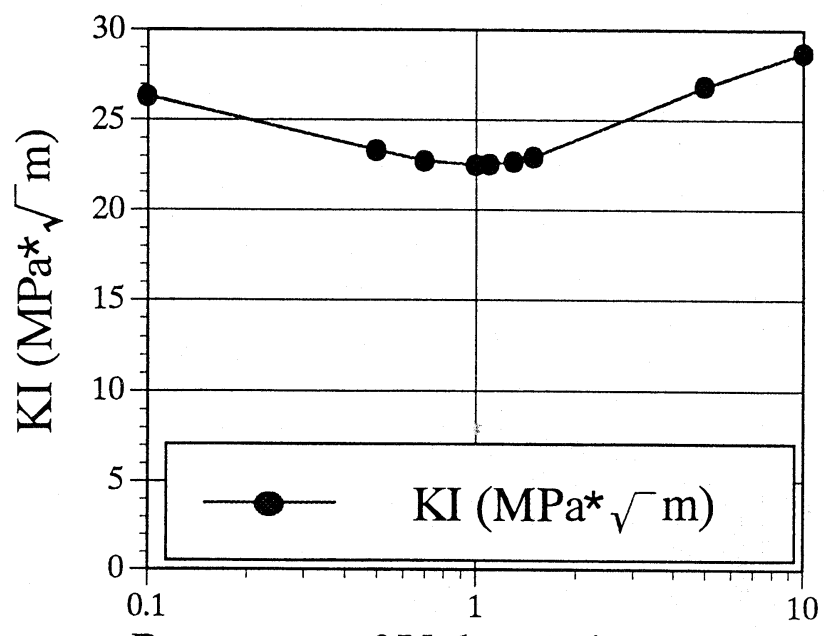

Parameter of Volumetrirc Ratio m

Figure 14 Maximum stress intensity factor versus $m$ 
mum stress intensity factor has minimum value. Then, the linear change of the composition should be selected.

\section{CONCLUSION}

(1) The thermal stresses in the plate of FGM can be largely decreased provided that the volumetric ratio of the composition of the ceramics and metal is pertinently selected.

(2) The transient thermal stress in the plate of the FGM can be solved by analytical method.

(3) The damage zone from the ceramics surface due to thermal shock during cooling stage is restricted within a very thin layer which is close to the surface.

(4) The crack-tip fields are the same as those in the homogeneous materials provided that the material properties of FGM are only continuous. The heat fluxes have a square root singularity at the crack tip. The elastic crack tip field is characterized by K-field of linear fracture mechanics.

(5) The unstable crack propagation occurs between $a_{i}=0.04 \mathrm{~mm}<a<0.1 \mathrm{~mm}$ at early time during cooling due to thermal shock, and after them a stable crack propagation occurs till a $=0.9 \mathrm{~mm}$.

(6) When the composition of the metal and ceramics changes linearly, the maximum stress intensity factor and maximum stress have minimum values.

Nomenclaure

\begin{tabular}{ll}
$\boldsymbol{c}$ & Specific heat \\
$\boldsymbol{E}$ & Young's modulus \\
$\boldsymbol{f}$ & Safty factor \\
$\boldsymbol{F}$ & Airy's stress function \\
$\boldsymbol{l}$ & Thickness of the plate \\
$\boldsymbol{P}$ & Porosity \\
$\boldsymbol{T}$ & Temperature \\
$\boldsymbol{T}_{\boldsymbol{a}}, T_{\boldsymbol{b}}$ & Given temperatures at boundary planes \\
$\boldsymbol{T}_{\boldsymbol{0}}$ & Initial temperature \\
$V_{m}$ & Volumetric ratio of the metal \\
$\boldsymbol{x}$ & Cartesian coordinate \\
$(\boldsymbol{r}, \theta)$ & Cylindrical coordinate \\
$\lambda$ & Thermal conductivity \\
$\lambda_{g}$ & Thermal conductivity of air \\
$\kappa(=\lambda / c \rho)$ & Thermal diffusivity \\
$\alpha$ & Linear thermal expansion \\
$\rho$ & Radius of cuavature and Density \\
$\varepsilon_{0}$ & Strain at $\boldsymbol{x}=\mathbf{0}$ \\
$\varepsilon_{i j}$ & Strain tensor \\
$\sigma$ & Stress \\
$\sigma_{i j}$ & Stress tensor \\
$\nu$ & Poisson's ratio \\
$\nabla^{2}$ & Laplacian operator \\
$\nabla$ & Gradient operator \\
\hline
\end{tabular}




\section{REFERENCES}

1) Yamanouchi, M, et al., (1990), Proceedings of the First Int. Sympo. on Functionally Gradient Materials.

2) Holt, J. B. et al., (1993), Functionally Gradient Materials, Ceramic Transaction Vol. 34, The Amsrican Ceramic Society.

3) Cubberly, W. H., (1989), Metals Handbook, 9th Ed., Vol. 3, ASM.

4) Touloukian, Y. S., (1973), Thermophysical Properties of Matter, Vol. 1, No. 2, IFI/Plenum.

5) Kingery, W. D., Bowen, H. H., and Uhlmann, D. R (1976), Introduction of ceramics, Jhone Wiley $\&$ Sons.

6) Kondo, R., (1986), Porous Materials, Gihoudo.

7) Noda, N., (1986), Thermal Stresses in Materials with Temperature-Dependent Properties, in R. B. Hetnarski(ed.), Thermal Stress I, Elsevier Science Publishers, Amsterdam, pp. 391-483.

8) Sih, G. C., (1965), Heat conduction in the infinite medium with lines of discontinuities, ASME, Journal of Heat Transfer, Vol. 87, pp. 293-298. 